\title{
CORRESPONDENCE
}

\section{FUSION AMPLITUDE WITH ABNORMAL RETINAL CORRESPONDENCE?}

\section{To the Editorial Committee of the British Journal of Ophthalmology}

SiRS,--In 1960 we suggested that the surgical treatment of squint at a very early age (squint, not mere paralysis uncomplicated by strabismic alterations) leads, in the great majority of fortunate cases, to small angles of anomaly (Arruga and Downey, 1960). We reached this conclusion by investigating a number of cases which were apparently cured when checked by classical methods, but which when investigated with more stringent methods showed minute angles of anomaly with little or no amplitude of fusion.

In true oculomotor palsy, especially of the vertical type (in purely horizontal cases the distinction between squint and paralysis or paresis is sometimes extremely difficult), the operation is of primary interest. This is true even when surgery is carried out at an age when accurate tests are impossible, if there are strong reasons to believe that strabismic alterations have not complicated the primary disturbance. If the surgical result is good the child may be left unoccluded. Furthermore, it is not infrequent to see a compensatory torticollis rectified in a few days, or even immediately.

In cases of squint, however, our results seem to confirm those of many surgeons who have verified their cases with exacting tests, and the theoretical reasons which condemn early operation as a sole treatment. Needless to say, if occlusion is maintained, however perfect the surgical result, there is no strong reason to oppose an early operation as a first step. But in this neighbourhood at least, it becomes far more difficult to persuade the parents to maintain occlusion when the eyes appear straight. Naturally, if the occlusion is not maintained zealously, the consequences will be much worse after a good surgical result.

The theoretical reasons, many of the essential points of which were stated by Bielschowsky about 50 years ago and have recently been reviewed and completed by Prof. C. Cüppers, can be summarized as follows:

(1) Post-operative parallelism of the eyes in the functional sense should be a sine qua non, and it can only be attained if an impulse of fusion is present.

(2) In squints of early onset, one cannot rely on a bi-foveal impulse of fusion. It is a common experience, even in recently-acquired paretic deviations, that amplitude of fusion can rapidly be lost under the influence of the deviated position.

(3) Where the normal sensory relationship is not well-founded, a harmonious correspondence is easily and rapidly achieved post-operatively with the slightest variation of the angle of deviation. Investigation with minimal dissociation tests, such as those of Tschermak and Bagolini, strongly support this view. In the case of apparent 100 per cent. post-operative parallelism, verified by the cover test (the only one possible to use in tiny children), one cannot guarantee that harmonious correspondence is being achieved at the objective angle of $0^{\circ}$ in all directions and at all distances. In the immediate post-operative period minute variations in the angle of deviation always occur.

(4) Whilst the possibilities of single binocular vision are remote if the child's eyes are left straight or slightly divergent (in a case of esotropia) after an early operation, they are nil if the eyes remain in a slightly convergent position. Oddly enough, some surgeons who advocate early operation also recommend a minimal under-correction (the ideal 
status to create small-angle abnormal retinal correspondence) ... "to prevent secondary divergence", but it must be remembered that the normal and physiological amplitude of divergence is limited and therefore more than difficult to develop in a case of squint originally convergent.

Hence it seems probable that, in the majority of cases (in our experience in practically all), "successful" early operation of true squint will lead to a small and incurable angle of anomaly. Because of this it is necessary to establish a normal retinal correspondence and a minimum of fusion amplitude pre-operatively. To this end, occlusion should be maintained until the child has reached an age which allows him to cooperate in the requisite orthoptic or pleoptic exercises. In certain circumstances exercises may be effective if started immediately after the operation if a thorough pre-operative investigation has been carried out (i.e. surgery may precede orthoptics).

Although our results qua binocular single vision, in private practice only, are below the 60 per cent. achieved at Giessen by Prof. Cüppers, they are much better than those we have seen after early operations performed both in this clinic and elsewhere.

Apparently some surgeons, according to Bridgeman (1961), "would prefer to obtain fusion amplitude with abnormal retinal correspondence" than "normal retinal correspondence with little or no fusion". We have found it very rare for patients with abnormal retinal correspondence to show any appreciable amplitude of fusion in space although angle changes may simulate this, particularly on the synoptophore. Latent normal correspondence, coexistent with an abnormal correspondence and dormant in daily life, may become manifest on the synoptophore when the tubes are moved towards the convergent position, thus causing bi-foveal stimulation and so simulating an amplitude of fusion.

As regards the second condition, it is often evident in cases of defective sensory Anlage. Normal retinal correspondence with little or no fusion may be seen in the orthophoric members of families including many strabismic subjects. Nevertheless, in many cases of squint, where adequate prophylaxis followed by successful orthoptic or pleoptic and surgical treatment has restored normal retinal correspondence, fusion (an acquired function) may be attained finally (as well as stereopsis) even if, in the early control examinations carried out after the surgery and post-operative orthoptics, it has seemed to be weak or almost absent.

Yours faithfully,

BARCELONA,

Alfredo Arruga, SPAIN.

RACHel A. DOWNeY.

August 12, 1961.

\section{REFERENCES}

ARruga, A., and Downey, R. (1960). Brit. J. Ophthal., 44, 492 (see bibliography). BrIDGEMAN, G. J. O. (1961). Ibid., 45, 318.

This correspondence is now closed.-Editor. 